\title{
INVESTIGACIONES
}

\section{Escritura colaborativa de textos argumentativos en inglés usando Google Drive*}

\author{
Collaborative writing of argumentative texts in English using Google Drive
}

\author{
Lucía Ubilla Rosales, ${ }^{a}$ Lilian Gómez Álvarez, ${ }^{b}$ Katia Sáez Carrillo ${ }^{c}$ \\ ${ }^{\text {a}}$ Facultad de Educación, Universidad Católica de Temuco \\ Telf.: (56) 94049911. Correo electrónico: lubilla@uct.cl \\ bepartamento de Idiomas Extranjeros, Facultad de Humanidades y Arte, Universidad de Concepción \\ Correo electrónico: ligomez@udec.cl \\ ${ }^{c}$ Facultad de Ciencias Físicas y Matemáticas, Universidad de Concepción \\ Correo electrónico: ksaez@udec.cl
}

\begin{abstract}
El objetivo de la presente investigación fue determinar el efecto de la escritura colaborativa en el desempeño de estudiantes de inglés como L2 al producir textos argumentativos en dicha lengua, apoyados por una herramienta para escritura colaborativa en línea (Google Drive) y un tratamiento de doce sesiones en modalidad semipresencial, y comparar los resultados con un grupo control que desarrolló los textos de manera individual. Se trata de un estudio cuasiexperimental con pretest, postest inmediato y dos postest diferidos para ambos grupos, con un modelo de efectos mixtos de medidas repetidas. Los resultados permiten validar la hipótesis de que la escritura colaborativa apoyada por herramientas tecnológicas favorece, facilita y agiliza el logro de los alumnos en la escritura en inglés como L2 en modalidad semipresencial y en contexto de instrucción con fines académicos.

Palabras clave: L2, CALL, modalidad semipresencial, instrucción con fines académicos.
\end{abstract}

\begin{abstract}
The objective of this research was twofold: to measure the effect of collaborative writing in the performance of students of English for writing argumentative texts in English as L2 by means of an online collaborative writing tool (Google Drive) for a period of 12 sessions in a b-Learning environment, and to compare these results with those of a control group working individually. The quasi experimental study involved pre-test, immediate post-test, and two delayed post-tests for both groups, in a mixed model for repeated measures. Results allow for hypothesis validation, namely, that b-Learning collaborative writing supported by technological tools improves, facilitates and encourages student gains in writing in English as L2 in an instructional environment for academic purposes.
\end{abstract}

Key words: L2, CALL, blended learning, instruction for academic purposes.

\footnotetext{
Tesis doctoral realizada en el marco del proyecto MECESUP USC-0805 "Implementación de propuesta de innovación curricular para la formación inicial de profesores de inglés basada en resultados de aprendizaje y competencias" de la Universidad Católica de la Santísima Concepción y la Universidad Católica de Temuco.
} 


\section{CONSIDERACIONES PREVIAS}

\subsection{APRENDIZAJE COLABORATIVO}

El aprendizaje colaborativo responde a un contexto sociocultural en el que se plantea que aprendemos socialmente y el aprendizaje es responsabilidad del estudiante, principio básico en la teoría sociocultural de Adquisición de Segundas Lenguas (ASL) y también en el tratamiento del proceso de expresión escrita. Este tipo de aprendizaje se centra, básicamente, en el diálogo y la negociación a través de la palabra, en que se colabora con el fin de lograr una meta común. Se da, entonces, un principio fundamental que dice relación con el rol que cumple la interacción social en el aprendizaje. Es así como, según Bruffee (1993) saber es "algo que construyen las personas hablando entre ellas y poniéndose de acuerdo" (p. 3). La premisa básica del aprendizaje colaborativo es la construcción del consenso a través de la cooperación de los miembros del grupo. Al mismo tiempo, en este tipo de aprendizaje se le atribuye mayor responsabilidad individual al estudiante y, por lo tanto, la estructura de la actividad tiende a ser más libre; además, parece existir mayor énfasis en el proceso. Es posible observar que el aprendizaje colaborativo cambia la responsabilidad del aprendizaje del profesor como experto al estudiante, y asume que el profesor es también un aprendiz (Bruffee, 1995).

Entre las principales características planteadas por diversos autores (Bruffee, 1993; Dillenbourg, 1999; Graham \& Misanchuk, 2004; Hilton \& Phillips, 2010; Klecker, 2003; Littleton \& Hakkinen, 1999; Meseke, Nafziger \& Meseke, 2008; North, Linley \& Hargreaves, 2000; Panitz, 1997; van der Laan Smith \& Spindle, 2007) en relación al trabajo colaborativo destacan las siguientes:

- debe haber un diseño intencional, esto es, las actividades deben estructurarse con un objetivo de aprendizaje intencional.

- debe existir colaboración real en la tarea por desarrollar, lo cual implica que todos los participantes del grupo estén activamente comprometidos en dicho trabajo, para así lograr la meta en relación con el o los objetivos comunes.

- debe haber un tipo de enseñanza significativa; básicamente, al trabajar colaborativamente, los aprendientes deben ampliar sus conocimientos o profundizar su comprensión respecto de un tema determinado.

- $\quad$ se debe centrar el trabajo en grupo hacia la elaboración en conjunto de un producto final, que es resultado del aporte de una parte del grupo o de su totalidad, y se evalúa por todos o la parcialidad de estos.

- $\quad$ se espera que los integrantes del grupo negocien las tareas, los roles y las responsabilidades.

- debe existir un compromiso de cada miembro del grupo para aprender algo a través del trabajo que se realizará colaborativamente; en consecuencia, la comunicación y la negociación son fundamentales, ya que deben llegar a acuerdos respecto de cómo realizarán la tarea, los procedimientos que adoptarán, la división del trabajo, las tareas por realizar, entre otros aspectos.

- es importante la interacción entre los miembros del grupo, una meta compartida, entendida y consensuada, respeto mutuo y confianza, comunicación continua, ambientes formales o informales, líneas claras de responsabilidad, autoridad compartida y responsabilidades individuales y grupales aceptadas.

- la selección de los integrantes en cada grupo puede seguir diversos criterios, a 
saber: por asignación al azar, por decisión de los estudiantes, por autoselección, o por selección del profesor.

- $\quad$ el tamaño del grupo puede variar; sin embargo, hay que tener presente que los grupos muy pequeños contienen menos diversidad y pueden carecer de estilos de pensamiento divergente y experiencia variada, lo cual ayuda a la toma de decisiones grupales; por otro lado, en los grupos más grandes es difícil asegurar la participación de todos los integrantes.

- $\quad$ respecto de los roles, se pueden definir como funciones o responsabilidades explícitas que guían el comportamiento individual y regulan la interacción grupal; asimismo, el establecimiento de roles promueve la cohesión del grupo, la responsabilidad individual y permite especificar las responsabilidades de cada integrante.

\subsection{ESCRITURA COLABORATIVA}

En uno de los primeros estudios de escritura colaborativa, Allen y otros (1987) establecen una definición para este tipo de escritura, caracterizándola como aquella en la cual un grupo de colaboradores produce un texto compartido y se involucra en una interacción sustantiva acerca del documento, compartiendo el poder y la responsabilidad en la toma de decisiones. La escritura colaborativa se focaliza en todo el proceso de escritura de un documento a través de un esfuerzo compartido. Es un proceso social en que los aprendientes enfrentan conflictos y deben negociar para alcanzar un consenso. El conflicto es la discusión entre dos o más personas, que indica desacuerdo respecto de sus objetivos, comportamiento, puntos de vista u opinión y que, sin lugar a dudas, puede ayudar a mejorar el trabajo colaborativo y ser beneficioso para el aprendiente, aunque en algunos casos puede también representar un peligro para el buen desempeño del trabajo, detalle no menor (Shantz, 1987; Tocalli-Beller, 2003). Además, ayuda a los estudiantes a desarrollar destrezas tanto en la planificación, en la confección de borradores, en la revisión periódica y colaborativa de los escritos, así como en el conocimiento del lenguaje, el contexto y el público meta (Derry, 1992).

Recientemente, se ha definido la escritura colaborativa como la producción en conjunto o la coautoría de un texto por dos o más autores (Storch, 2011).

Por otro lado, la escritura colaborativa tiene como característica principal que los aprendientes no solo realizan una subdivisión de las tareas y componen individualmente sus propios textos, sino que también comparten derechos de autor, leen los textos escritos por sus compañeros y adoptan el rol de coescritores o coeditores, ayudándose así unos a otros para elaborar textos de mejor calidad. Igualmente, este tipo de escritura ayuda a los alumnos a desarrollar estrategias para entregar feedback a sus pares, ya que revisar y evaluar los textos de sus compañeros es parte del proceso que desarrollan (De Graaff, Jauregi \& Nieuwenhuijsen, 2002; Jauregi, Nieuwenhuijsen \& de Graaff, 2003).

En cuanto a las ventajas de la escritura colaborativa, es importante considerar: 1) los beneficios afectivos para el estudiante y el grupo, ya que este tipo de trabajo reduce la ansiedad, aumenta la interacción, la confianza en sí mismos, la autoestima y provoca un aumento de la motivación; 2) la creación de un ambiente de trabajo relajado y distendido en el aula, debido al intercambio comunicativo que es propio en esta clase de actividades, y 3) la existencia de una audiencia auténtica, lo que permite a los aprendices la oportunidad de conocer lo que los lectores comprenden y lo que no entienden de sus escritos (Johnson \& Johnson, 1999; Raimes, 1998; Reid \& Powers, 1993; Rollinson, 2005). 
Muchos investigadores plantean que, tanto en lengua materna como en segunda lengua, existen evidencias de que esta metodología contribuye a mejorar la calidad de la escritura (Storch, 2005); al mismo tiempo, ayuda a que quienes escriben sean más conscientes de aquellos para quienes escriben (Leki, 1993); aumenta la motivación por parte de los aprendientes y, además, ayuda a que los alumnos pongan más atención a las estructuras del discurso, así como también a la gramática y uso del vocabulario (Kowal \& Swain, 1994; Swain \& Lapkin 1998).

Entre otros de los hallazgos realizados en esta área, se ha descubierto que la escritura colaborativa y el trabajo en equipo permiten a los escritores novatos practicar estilos constructivos de comunicación. Esto, a su vez, ayuda a que produzcan textos con mayor coherencia y cohesión, redacten sus ideas de manera más creativa y utilicen un repertorio lingüístico más amplio que aquellos textos elaborados individualmente (Fernández, 2009; Fisher et al., 2010; Guzmán, 2007; Littleton, Miell \& Faulkner, 2004; Rojas-Drummond, Albarrán \& Littleton, 2008)

En resumen, los diversos estudios que se han realizado en torno a la escritura colaborativa sugieren que el impacto de los factores afectivos al trabajar en grupo, inevitablemente influirá de forma positiva en los resultados. Por otra parte, en relación con aspectos más académicos, se ha demostrado que la escritura colaborativa sí posee beneficios respecto de la precisión que logran los aprendientes en la construcción del texto, especialmente en estudiantes que están en o por sobre el nivel intermedio.

\subsection{TECNOLOGÍA Y DESARROLLO DE LA EXPRESIÓN ESCRITA EN L2}

El nuevo paradigma tecnológico en el que estamos inmersos ha permitido la proliferación de diversas tecnologías que potencian el trabajo colaborativo, como los blogs, wikis, redes sociales, feeds, Delicious, Skype, Moodle, Slideshare, Youtube, Google Drive (ex Google Docs), entre otras. Lam y Pennington (1995) ya planteaban que las nuevas tecnologías entregarían beneficios positivos para el trabajo de escritura colaborativa, ya que permitirían una revisión y feedback más conveniente, con un tiempo de respuesta más rápido y un aumento de la motivación y la creatividad, además de la posibilidad de trabajar simultáneamente desde cualquier punto geográfico.

Es así como investigaciones actuales en escritura con uso de la Web 2.0 han demostrado que estas tecnologías realmente entregan nuevas oportunidades a los aprendientes para involucrarse en el proceso de escritura y mostrar sus productos terminados. Además, se han detectado mejoras con relación a la fluidez, la precisión y la valorización que los alumnos otorgan a la oportunidad de compartir feedback con sus pares (Elola \& Oskoz, 2010; Hoopingarner, 2009; Ware \& O’Dowd, 2008). Sin embargo, también se ha demostrado que los alumnos se focalizan más en el significado que en la forma y que este tipo de tecnologías modernas contribuye a que los aprendientes desarrollen un sentido de pertenencia hacia el texto que construyen en conjunto, desarrollando, a la vez, un cierto grado de autonomía respecto del profesor (Kessler, 2009).

Así, estos recursos han empezado a usarse en aula, ya que generan espacios de comunicación aptos para el desarrollo de las habilidades comunicativas y, especialmente, actitudes de un nuevo tipo de alfabetización tecnológica crítica, colaborativa y creativa. Del mismo modo, estas herramientas permiten crear un entorno práctico eficiente para la socialización y la culturización de los aprendientes (Bruns \& Humphreys, 2005; Esteve, 2009; Pérez, 2008). 
Muchas de estas aplicaciones de la web 2.0 permiten, además, que los aprendientes interactúen de manera sincrónica o asíncrona, ya sea con sus compañeros, profesores o consigo mismos en relación con sus propios trabajos y de los demás. Este es el caso de Google Drive (edición educacional de Google Apps), herramienta gratuita, creada especialmente para escuelas, universidades y organizaciones sin fines de lucro y que no requiere instalación de software. Esta aplicación facilita el trabajo individual y fortalece el trabajo colaborativo, ya que ofrece la posibilidad de compartir un documento con otros, solo para visualizarlo, para colaborar en su construcción o para publicarlo en la web.

Además, permite guardar distintas versiones de un documento para, así, tener un registro de dichas versiones. El aprendiente puede realizar una revisión de aspectos de redacción e interactuar casi inmediatamente con sus compañeros o profesor y recibir sus opiniones respecto del texto en cuestión. Es decir, esta herramienta favorece el desarrollo del trabajo sincrónico, ya que los estudiantes pueden trabajar de manera simultánea en un mismo documento, desde diferentes puntos geográficos, distinguiéndola de otras herramientas como las wikis (Brown \& Adler, 2008; Conner, 2008; Lozano et al., 2011; Oishi, 2007; Tharp, 2010).

Otra de las ventajas de Google Drive es que provee un mecanismo de monitoreo para ver la participación de los estudiantes y la forma en que plasman sus ideas en un mismo documento. El hecho de tener acceso al documento vía internet desde la casa o desde cualquier lugar remoto y trabajar en este en tiempo real aumenta la eficacia de la comunicación y, por ende, el beneficio académico (Kieser \& Ortiz Golden, 2009; Tharp, 2010).

Como lo establece García (2010), Google Drive beneficia la escritura colaborativa y, adicionalmente, incluye enlaces a una serie de herramientas que ayudan al aprendiente a mejorar su producción escrita, tales como un tesauro o un diccionario. Una vez que el documento es compartido a través de la red, el profesor $u$ otro aprendiente pueden acceder a la producción e introducir las correcciones para mejorar el texto, marcando claramente la autoría de la modificación y las mejoras producidas en distintos colores. Así, la aplicación permite al docente controlar también el trabajo individual de sus alumnos y da a cada uno de ellos la seguridad de que lo que han escrito puede ser claramente identificado.

Del mismo modo, Pico y Rodríguez (2012) destacan el hecho de que los documentos en Google Drive pueden ser compartidos de diferentes formas: publicar un archivo en internet, invitar a otros a trabajar en un archivo, participar en la edición de archivos que otros generaron e interactuar en tiempo real con otros usuarios, sobre un mismo documento. La herramienta permite, además, la opción de trabajar sin conexión a internet, por momentos.

\section{METODOLOGÍA}

\subsection{OBJETIVOS}

- Determinar el efecto del trabajo de escritura colaborativa en el desempeño de estudiantes de Pedagogía en Inglés, en la producción de textos argumentativos en L2, apoyados por una herramienta de escritura colaborativa en línea (Google Drive) en modalidad semipresencial.

- Comparar los resultados en el desempeño de estudiantes de nivel B2 de competencia en inglés como L2 en la producción de textos argumentativos, utilizando una 
metodología de escritura colaborativa y una metodología de escritura individual, apoyados por una herramienta para escritura colaborativa en línea (Google Drive), en modalidad semipresencial.

\subsection{TIPO DE INVESTIGACIÓN}

El presente estudio utilizó un modelo de investigación cuantitativa basada en un diseño cuasiexperimental de clases intactas, en el que los grupos control y experimental estaban previamente conformados. El grupo experimental G1 trabajó con escritura colaborativa $(\mathrm{N}=18)$ y el grupo control G0 trabajó con escritura individual $(\mathrm{N}=15)$. Ambos grupos utilizaron la herramienta Google Drive y una modalidad semipresencial. Las clases intactas correspondieron a dos secciones pertenecientes a un curso de último año (cuarto) de lengua inglesa para estudiantes de pedagogía en inglés durante el 2 do semestre de clases. Los alumnos de este curso tienen un nivel de proficiencia B2 en inglés, según el Marco Común Europeo de Referencia (Consejo de Europa, 2002).

Asimismo, el modelo contempló un pretest, un postest inmediato y dos postest diferidos para el grupo experimental y el grupo control, y un tratamiento que duró doce sesiones (seis presenciales y seis no presenciales).

Los cuatro test aplicados consistieron en el desarrollo de un texto escrito en formato de texto argumentativo en inglés, según los requerimientos de la tarea 2 del examen internacional IELTS. Estos test se administraron antes del tratamiento, inmediatamente después y en forma diferida. Al mismo tiempo, los dos grupos completaron un cuestionario inicial y un cuestionario de percepciones.

\subsection{MUESTRA}

La muestra fue de tipo no probabilística intencionada constituida por 33 sujetos, pertenecientes a las dos secciones del último curso de lengua inglesa de la carrera de pedagogía en inglés de una universidad regional. Dicha asignatura es de carácter obligatorio y consta de seis horas cronológicas semanales. De acuerdo con los resultados del cuestionario aplicado antes de comenzar la intervención, se puede establecer lo siguiente: que el rango de edades de los participantes de la muestra fluctuaba entre los 21 y 24 años; que $76 \%$ correspondía a sexo femenino y $24 \%$, al masculino ( 25 mujeres y 8 hombres); y que el nivel de competencia lingüística de entrada de los alumnos era intermedio avanzado, equivalente al nivel B2 del MCER, evaluado sobre la base de una prueba de FCE mock test administrada al finalizar el curso anterior. Para efectos de la investigación, se asignó de manera aleatoria a los alumnos de la sección 1 de la asignatura al grupo experimental $(\mathrm{G} 1, \mathrm{~N}=18)$ y a los estudiantes de la sección 2, al grupo control (G0, N=15). Ambos grupos tuvieron a la misma profesora de inglés (hablante nativa), quien ya había trabajado con dicho nivel en años anteriores. De esta forma, se evitó que la investigadora pudiera ser considerada como un factor interviniente en los resultados.

\subsection{TRATAMIENTO}

Tanto el grupo experimental como el grupo control, después del pretest, trabajaron con los contenidos establecidos en el programa del curso de lengua inglesa, sobre la base del módulo 
de intervención confeccionado para esta investigación. Dicho módulo constaba de 12 sesiones (cada sesión era de 1,5 horas por semana). Del total de sesiones, seis se desarrollaron en la modalidad presencial y seis, en la modalidad no presencial. Las tareas asignadas para las clases presenciales y no presenciales en el grupo experimental se realizaron con una metodología de trabajo de escritura colaborativa, apoyados por la herramienta de la web 2.0 Google Drive, complementada por un blog elaborado para efectos de la investigación. El grupo control, por su parte, llevó a cabo las mismas tareas con las mismas herramientas pero con una metodología de escritura individual. Al finalizar el proceso, a ambos grupos se les aplicó un postest inmediato. Transcurridos cuatro meses, se aplicó el postest diferido 1, y transcurridos otros cuatro meses, se aplicó el postest diferido 2 .

\subsection{INSTRUMENTOS}

Se utilizaron varios instrumentos para recolectar los datos necesarios para responder las preguntas de investigación del estudio, principalmente mediante el uso de fuentes primarias, es decir, los estudiantes proporcionaron directamente la información que se analizó posteriormente. Esta recolección se efectuó antes de iniciar el módulo de intervención, con la encuesta de intereses, el cuestionario inicial y el pretest y, al final del proceso, con el postest inmediato, postest diferido 1 y postest diferido 2 .

\subsection{IMPLEMENTACIÓN DEL MÓDULO}

Como se planteó anteriormente, las temáticas, los objetivos comunicativos y los contenidos gramaticales que se trabajaron en el módulo estaban en directa relación con los contenidos del programa del curso lengua inglesa.

Los seis grupos que se conformaron en el grupo experimental escribieron los mismos tipos de textos (argumentativos), aunque el proceso se dio de forma paulatina. El primer texto desarrollado (sesión 1 y 2) fue la introducción de un texto argumentativo. El segundo texto (sesiones 3 y 4) consistió en la introducción y el cuerpo de un texto argumentativo. El tercer texto (sesiones 5 y 6) consistió en la redacción del primer texto completo, introducción, cuerpo y conclusión. Posteriormente, los estudiantes desarrollaron tres textos argumentativos completos. El procedimiento adoptado para la realización de las tareas escritas incluyó pretareas, tareas de proceso y postareas. En este proceso destacan: 1) discutir aspectos específicos sobre un texto argumentativo, por ejemplo: organización de argumentos, diferencias entre hechos y opiniones, diversas fuentes para respaldar argumentos; 2) observar tres videos breves (de YouTube) sobre diferentes temáticas para elegir el tema del ensayo y las instrucciones para desarrollarlo; abrir un archivo en Google Drive (Archivo 1) para registrar las ideas de la discusión del tema; elegir un color de fuente distinto para cada rol del proceso de escritura; organizar las ideas en formato de guion (outline); 3) sobre la base del boceto inicial, buscar información extra acerca del tema para apoyar sus argumentos, con uso de enlaces sugeridos en el blog; abrir un segundo archivo (Archivo 2) en Google Drive para escribir el primer borrador (introducción, cuerpo y conclusión). Aunque esto era responsabilidad de un miembro del grupo (drafter), los demás estudiantes podían agregar ideas, las que se registraban según el color de fuente elegido previamente; 4) el revisor verificaba el tema, la tesis, los argumentos y la conclusión del texto redactado. Los demás podían ofrecer comentarios que quedaban registrados en los colores elegidos. El editor 
revisaba las estructuras, la puntuación y la ortografía. Después, el revisor escribía el texto corregido en un nuevo archivo de Google Drive (Archivo 3), entre otras.

\section{RESULTADOS Y ANÁLISIS DE DATOS}

Los resultados obtenidos a través de las evaluaciones y cuestionarios fueron incorporados en una base de datos que se analizó utilizando el software R v3.1.3. Para cada uno de los resultados y para el puntaje total se ajustó un modelo de efectos mixtos de medidas repetidas. En todos los ajustes se consideraron como efectos aleatorios los participantes del estudio, como variables de efectos fijos el tipo intervención (Control: intervención tradicional "trabajo de escritura individual", Experimental: intervención impartida "trabajo de escritura colaborativa") y los momentos en que se realizaron las evaluaciones (antes y después de la intervención y las dos pruebas diferidas). Con la finalidad de controlar posibles efectos intervinientes, se analizó la incorporación de covariables (edad, promedio de notas del curso anterior, etc.) las cuales finalmente no fueron consideradas, ya que no se encontró evidencia estadísticamente significativa para su inclusión.

$\mathrm{Al}$ inicio del estudio, se realizaron diferentes pruebas a ciertas variables para determinar la equivalencia entre las dos secciones del curso de lengua inglesa. Es así como el test de Mann-Whitney se utilizó para comparar la edad de los estudiantes y el promedio de notas con el que ingresaron los alumnos al comienzo del semestre, y el test exacto de Fisher para determinar si la distribución en ambos grupos era la misma respecto de las variables sexo, años de estudio en la carrera, uso de internet, uso de Google Drive y frecuencia de uso.

Para las variables numéricas se utilizó el test de Shapiro-Wilk para verificar el supuesto de normalidad. En todos los test se utilizó un nivel de significancia del 0,05; esto es, cada vez que el valor $\mathrm{p}$ de un test fue menor que 0,05 , se consideró que había significancia estadística.

\subsection{ANÁLISIS PRE Y POSTEST INMEDIATO EN AMBOS GRUPOS}

A continuación se muestran los resultados del grupo de control y del experimental en las pruebas realizadas, con la finalidad de obtener, por un lado, el estado inicial de cada grupo, previo al ciclo de exposición al módulo de intervención lingüística en que se trabajó en una metodología de escritura colaborativa y, por otro, una visión global en las pruebas posteriores de la incidencia del trabajo de escritura colaborativa en las tareas realizadas.

Con el fin de comparar las respuestas de los grupos control y experimental, se ajustó un modelo mixto de medidas repetidas, ya que son evaluados los mismos sujetos en el pretest y en el postest. Las variables independientes fueron grupo (control-experimental), rendimiento en pruebas de producción de textos argumentativos (pretest, postest, postest diferido 1 y postest diferido 2) y sujeto, este último de efecto aleatorio.

A continuación se muestran los resultados de medias y desviaciones estándar para ambos grupos en todos los test aplicados. 
Tabla 1. Medias y desviaciones estándar por grupo en pre y postest

\begin{tabular}{|l|c|c|c|c|}
\hline & \multicolumn{2}{|c|}{$\begin{array}{c}\text { Grupo Control } \\
(\mathrm{n}=15)\end{array}$} & \multicolumn{2}{c|}{$\begin{array}{c}\text { Grupo Experimental } \\
(\mathrm{n}=18)\end{array}$} \\
\hline & Media & D.E. & Media & D.E. \\
\hline Pretest & 18,5 & 2,0 & 16,6 & 2,9 \\
\hline Postest Inmediato & 21,6 & 1,4 & 21,9 & 2,3 \\
\hline Postest Diferido 1 & 21,2 & 1,3 & 21,5 & 1,6 \\
\hline Postest Diferido 2 & 20,3 & 0,9 & 21,0 & 1,5 \\
\hline
\end{tabular}

En la Tabla 1 se observa que en el pretest los resultados para el grupo experimental fueron más bajos que para el grupo control y que estuvieron menos concentrados alrededor de la media. Sin embargo, después de ser sometidos a la metodología de escritura colaborativa (postest), los resultados del grupo experimental mejoraron sustantivamente y se concentraron más alrededor de la media. Los puntajes se mantuvieron en el largo plazo (postest diferido 1 y 2) y continuaron manteniéndose en torno a la media. Además, se puede observar que ambos grupos obtuvieron resultados significativamente mayores en el postest inmediato comparados con los resultados obtenidos en el pretest (Grupo control: $\mathrm{M}=18,5$; $\mathrm{DE}=2,0)(\mathrm{p}=0,0004)$ y (Grupo experimental: $\mathrm{M}=16,6 ; \mathrm{DE}=2,9)(\mathrm{P}<0,0001)$ (Figura 1). Sin embargo, al comparar el aumento obtenido por cada grupo entre el pretest y el postest inmediato, se observa que el aumento del grupo experimental fue estadísticamente mayor (Figura 2) en el desempeño en la producción del texto argumentativo.

Figura 1. Comparación de las diferencias en rendimiento antes y después de la intervención

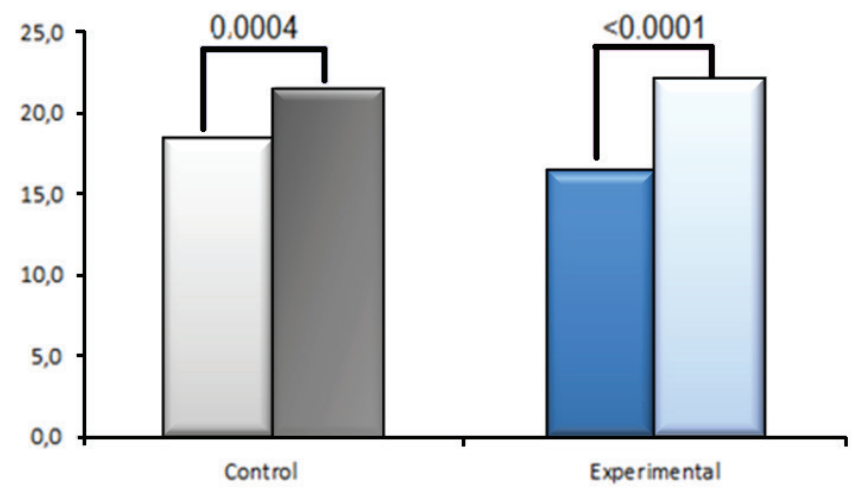


Figura 2. Comparación en aumento pretest y postest inmediato

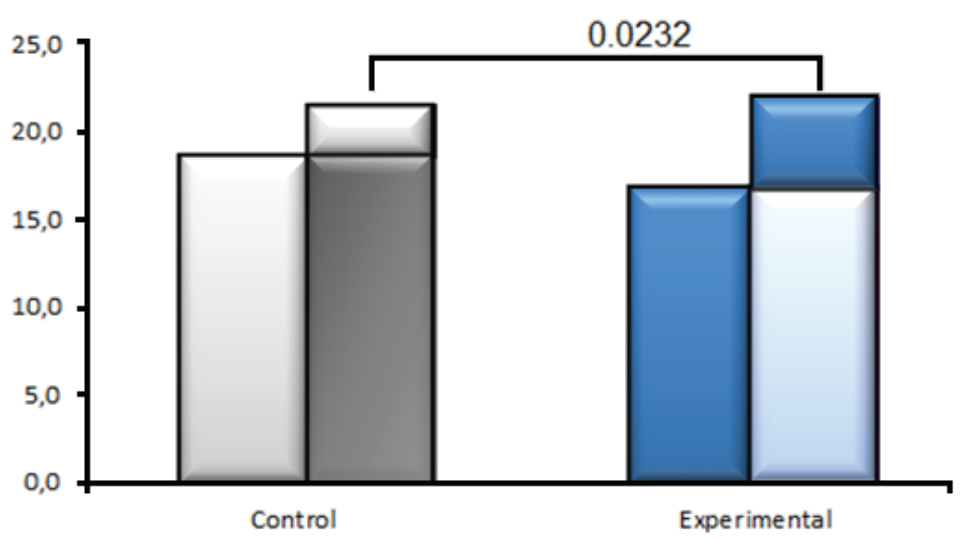

Estos resultados validan la hipótesis general del estudio, a saber: el trabajo de escritura colaborativa mejora el desempeño de estudiantes de inglés de nivel B2 en la producción de textos argumentativos en inglés como L2, apoyados por una herramienta para escritura colaborativa en línea (Google Drive) en modalidad semipresencial y en contexto académico de instrucción universitaria, ya que las diferencias en el desempeño de la producción de los textos solicitados respecto del grupo control son altamente significativas; es decir, el grupo experimental obtuvo una ganancia en su rendimiento notoriamente superior al del grupo de control en la escritura de textos argumentativos en inglés. Por lo tanto, los datos permiten sostener que fomentar el trabajo de escritura colaborativa mejora el desempeño en la producción de textos argumentativos en L2 (Guzmán \& Rojas-Drummond, 2012; Rojas-Drummond et al., 2010; Storch, 1999, 2005; Storch \& Wigglesworth, 2007).

\subsection{ANÁLISIS DE TEST DIFERIDOS EN AMBOS GRUPOS}

Luego de llevar a cabo el proceso de intervención lingüística en 12 sesiones, se aplicaron tres pruebas: un postest inmediato (noviembre), un postest diferido 1 (marzo) y un postest diferido 2 (julio) en ambos grupos, todo con el objetivo de medir el efecto de la aplicación de una metodología de escritura colaborativa para mejorar el desempeño en la producción de textos argumentativos en inglés, en el largo plazo.

A continuación, en la Figura 3, se presentan los resultados de las cuatro pruebas aplicadas (pretest, postest inmediato, postest diferido 1 y postest diferido 2) durante la investigación. 
Figura 3. Diferencias entre grupo control y experimental en todos los test

\section{Grupō Control}

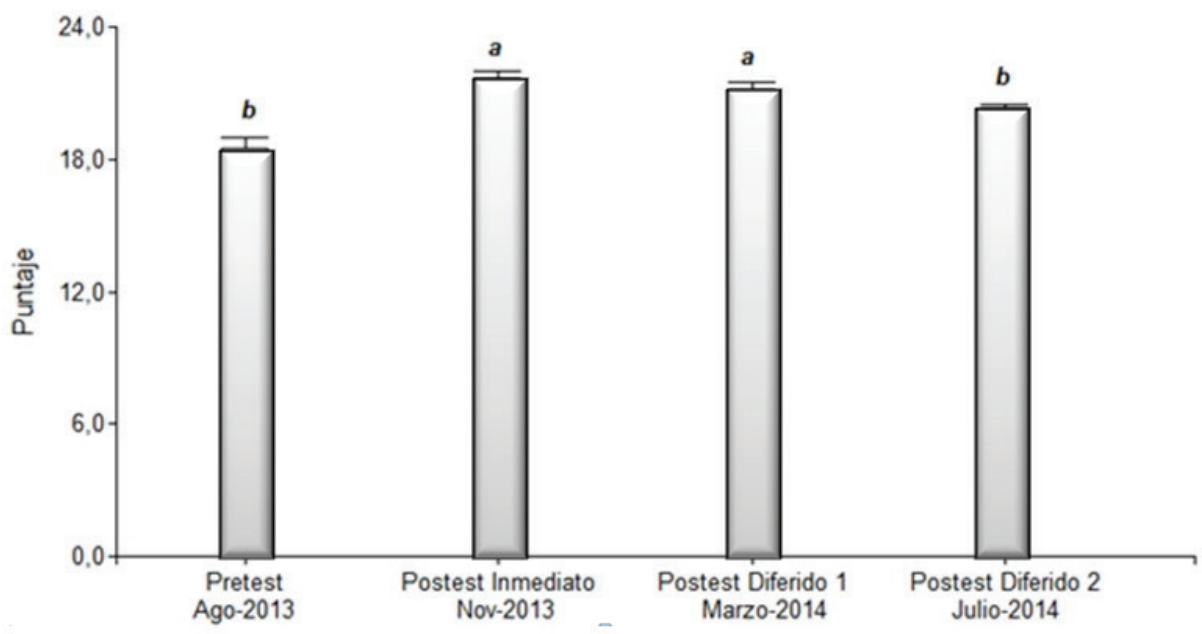

Grupo Experimental

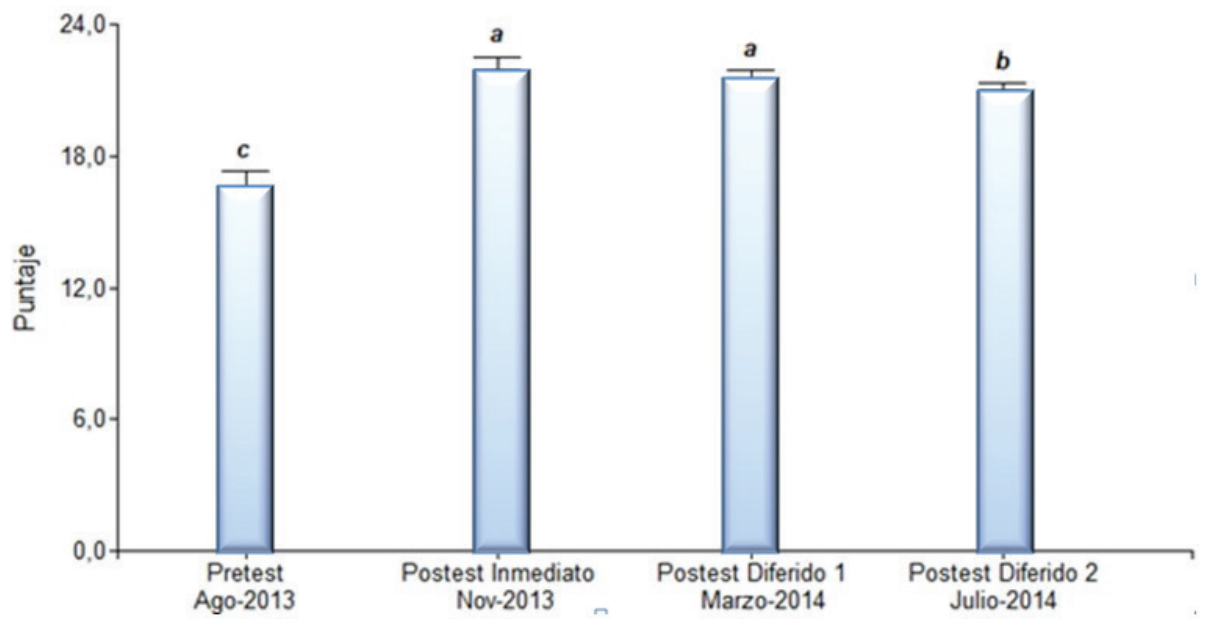

*Medias con letra distinta indican diferencia estadísticamente significativa $(\mathrm{p}<0,05)$.

Al comparar los resultados del postest inmediato (noviembre) y el postest diferido 1 (marzo), se observa que existe una disminución en el nivel de desempeño de la producción de los textos argumentativos; sin embargo, esta no es estadísticamente significativa tanto para el grupo de control como en el experimental. Es decir, la distancia entre el resultado del grupo experimental sigue estando a su favor, a pesar del tiempo transcurrido. 
Asimismo, al observar la cuarta evaluación, el postest diferido 2 (julio), se observa que ocurre una disminución significativa en cuanto al desempeño de la producción de los textos respecto del postest inmediato y del postest diferido 1, en ambos grupos. Entonces, es importante destacar que en esta última evaluación (postest diferido 2) los resultados del grupo control no difieren significativamente del promedio obtenido en el pretest, mientras que en el grupo experimental, aunque el promedio del postest diferido 2 baja, el resultado es superior al valor obtenido en el pretest. Este hecho muestra nuevamente que el grupo experimental obtuvo una ganancia en su rendimiento notoriamente superior al del grupo de control en la escritura de textos argumentativos. En otras palabras, el grupo control olvida más rápido o se acaba más rápido el efecto de la instrucción.

\section{DISCUSIÓN DE LOS RESULTADOS}

La primera pregunta de investigación intentaba averiguar el impacto del trabajo de escritura colaborativa en L2 en el desempeño de la producción de un texto argumentativo en estudiantes de pedagogía en inglés con un nivel B2 de acuerdo al Marco Común Europeo de Referencia para las Lenguas, apoyados por una herramienta que permite la escritura colaborativa en línea, como lo es Google Drive, en una modalidad semipresencial. La segunda pregunta pretendía dilucidar si es posible mejorar el desempeño de la producción de este tipo de texto.

Esta pregunta ha sido respondida examinando los resultados de los test de producción escrita, que muestran que los alumnos cuyo trabajo utilizó una metodología de escritura colaborativa tuvieron un desempeño significativamente superior en los textos producidos, comparados con el grupo de alumnos que trabajaron en una modalidad de escritura individual. Desde una perspectiva teórica, los resultados del postest en el grupo experimental se fundamentan en diversas investigaciones que refuerzan el hecho de que los alumnos que practican un tipo de escritura colaborativa a lo largo de una intervención son capaces de desarrollar paulatinamente las habilidades para producir textos coherentes de forma individual, autorregulada y autónoma. La escritura colaborativa, en consecuencia, mejora el desempeño de los alumnos en la producción del tipo de texto que se trabaje, haciéndolos más precisos (Guzmán \& Rojas-Drummond, 2012; Storch \& Wigglesworth, 2007).

Igualmente, este tipo de metodología permite a los alumnos participar en actividades significativas de escritura colaborativa, en las cuales interactúan y se comunican en pequeños grupos (pares o tríadas) mientras leen y escriben textos de tipo argumentativo. Este tipo de interacción permite que los alumnos desarrollen interrelaciones en áreas de tipo social y cognoscitivo del desarrollo dentro de la perspectiva sociocultural. Según Rogoff (2003), el conocimiento compartido socialmente se reconstruye y se convierte en algo cualitativamente nuevo. En este sentido, los textos desarrollados por los alumnos que participaron en el experimento fueron compartidos por una audiencia real, la cual estaba no solo interesada en leer dichos textos, entregando, pidiendo opinión y negociando puntos de vista, sino que también planteaba propuestas y proponía reformulaciones, lo que permitía elaborar textos de mejor calidad (Rojas-Drummond et al., 2010).

Por añadidura, es posible plantear que las tareas de escritura colaborativa en las cuales los alumnos construyen sus textos les permiten desarrollar un tipo de comunicación significativa y además se pueden comprometer en procesos cognitivos que ayudan a 
desarrollar la adquisición de la L2 (Kuiken \& Vedder, 2002). En otras palabras, no solo les ayuda a producir mejores textos en las evaluaciones de postest, sino que también estas tareas son una fuente de aprendizaje para el desarrollo de la adquisición de la L2. En este sentido, la investigación en escritura colaborativa, tanto en lengua materna (L1) como segunda lengua (L2), ha mostrado que este enfoque pedagógico tiene gran potencial, ya que requiere pensamiento reflexivo, ayuda a los aprendientes a focalizarse en la gramática, el vocabulario y el discurso, y además ayuda a reunir conocimiento acerca de la lengua (DiCamilla \& Anton, 1997; Donato, 1994; Hirvela, 1999; Storch, 2002; Swain \& Lapkin, 1998).

No obstante lo anterior, en el escenario nacional se observa que los docentes de inglés en Chile no dedican mucho tiempo al desarrollo de la escritura en sus clases, aunque al revisar los Planes y Programas de Educación se declara la importancia de desarrollar todas las habilidades en una segunda lengua. Se plantea, además, como lo evidencia la teoría, que la expresión escrita ofrece la posibilidad de expresar mensajes significativos en el idioma en forma comunicativa y contextualizada. Es así como, la escritura en inglés deja de considerarse como un medio para repasar patrones gramaticales o vocabulario en forma repetitiva y se convierte en una posibilidad de usar el idioma para comunicarse en situaciones reales. La enseñanza de la escritura contempla, además, instrucción sobre cómo generar ideas, cómo organizarlas y cómo conferirles coherencia, de acuerdo con el tipo de texto y el uso de expresiones y la gramática adecuadas. Por otro lado, los planes y programas de educación también declaran la importancia de utilizar técnicas colaborativas para el desarrollo del aprendizaje y el aprovechamiento de las diversas tecnologías para la comunicación.

Como se puede observar, entonces, tanto la teoría como los planteamientos del Ministerio de Educación (MINEDUC), diversas investigaciones y este estudio en concreto sustentan el hecho del impacto positivo que tiene el trabajo de escritura colaborativa en L2 en el desempeño de la producción de un texto en inglés, particularmente en este caso, textos argumentativos. Si bien esta investigación se llevó a cabo en un contexto universitario de aprendizaje del inglés como L2, no existirían impedimentos para realizar trabajos similares en otros contextos de aprendizaje, en otros niveles de competencia lingüística y en otros idiomas.

\section{LIMITACIONES}

Si bien es cierto que en el desarrollo de este estudio se cautelaron al máximo las condiciones de igualdad entre los dos grupos, salvo la variable que se estaba controlando, escritura colaborativa, es importante tener presente que trabajar con una metodología colaborativa requiere una planificación detallada de los objetivos, las tareas asignadas a los aprendientes, los roles por asignar, los materiales por utilizar y además los alumnos deben tener cierta expertise en el desarrollo de tareas de escritura colaborativa.

Asimismo, el factor relaciones humanas es algo muy difícil de controlar en cualquier tipo de estudio. En esta investigación se optó por la estrategia de autoselección de los integrantes del grupo ya que según diversos trabajos previos presenta beneficios en relación con la mejora en resultados académicos y trabajos de mayor nivel; menores dificultades para organizar sus horarios, ya que se conocen, y esto permite un trabajo más conducente; y disposición a comenzar el trabajo de forma inmediata (Graham \& Misanchuk, 2004; Hilton \& Phillips, 2010; van der Laan Smith \& Spindle, 2007). Sin embargo, de igual 
forma hubo grupos que tuvieron dificultades para organizarse con la asignación de las tareas y los roles. Afortunadamente lograron superar sus problemas y desarrollaron las tareas solicitadas de buena forma, pero las relaciones humanas es un factor importante a considerar en un estudio de este tipo.

Otro aspecto por considerar es el hecho de que hay personas que prefieren trabajar solas. En este estudio los alumnos del grupo experimental no manifestaron ningún problema con la idea de trabajar en grupo, pero en otros grupos humanos puede ocurrir que haya sujetos que no deseen trabajar colaborativamente; por lo tanto, los profesores deben estar conscientes de esta situación y dar la libertad a los alumnos que prefieren la modalidad individual, ya que, definitivamente, forzar una metodología de trabajo puede tornarse perjudicial para el trabajo final. En estos casos, se sugiere respetar el estilo de aprendizaje de cada alumno, pero también es importante mostrar a los alumnos los beneficios de este tipo de trabajo, pues al ser parte de una comunidad el trabajo colaborativo es fundamental en toda índole de actividades sociales.

En los últimos años ha aumentado el número de herramientas colaborativas en la web 2.0, pero para esta investigación solo se consideró Google Drive, y un tipo particular de texto (argumentativo). Sería interesante realizar otros estudios similares con otro tipo de texto y otro tipo de herramienta tecnológica, para así comparar resultados y ver cómo estas herramientas pueden ayudar de mejor forma a que los alumnos desarrollen sus habilidades de escritura y a mejorar la calidad del trabajo de los profesores al focalizarse en esta habilidad en particular.

Finalmente, si bien es cierto los resultados obtenidos en este estudio son valiosos para una variedad de contextos educativos, especialmente donde los aprendientes tienen un nivel avanzado de dominio de la L2, hay que tener presente que existen realidades escolares muy vulnerables cuyos niveles de adquisición de Inglés no logran los estándares propuestos a nivel país. Es por esto que sería un desafío interesante aplicar un estudio de características similares en los contextos antes mencionados, analizar los resultados, realizar las modificaciones necesarias e implementar nuevamente con el objetivo de ayudar a los alumnos de realidades más vulnerables a desarrollar habilidades de escritura en la L2 y así mejorar los niveles de Inglés al menos a nivel regional.

\section{CONCLUSIONES}

En respuesta al objetivo 1: "determinar el efecto del trabajo de escritura colaborativa en el desempeño de estudiantes de pedagogía en inglés en la producción de un texto argumentativo en inglés como L2, apoyados por una herramienta para escritura colaborativa en línea (como Google Drive) en una modalidad semipresencial" y al objetivo 2: "comparar los resultados en el desempeño de estudiantes de pedagogía en inglés en la producción de un texto argumentativo en inglés como L2, a través del uso de una metodología de escritura colaborativa y una metodología de escritura individual, apoyados por una herramienta para escritura colaborativa en línea (como Google Drive) en una modalidad semipresencial", se puede llegar a las siguientes conclusiones:

Se evidencia un efecto positivo del trabajo de escritura colaborativa de textos argumentativos en inglés mediado por la tecnología, ya que quienes escriben tienen la oportunidad de consultar su conocimiento explícito a sus pares y luego tomar decisiones 
más dialogadas en relación con el texto que se está construyendo. Esta es una forma más efectiva de crear nuevo conocimiento, al trabajar de forma individual, principalmente debido a que la colaboración involucra la obtención del conocimiento de diversas fuentes. Toda esta interacción que se produce en este tipo de trabajo facilita y ayuda enormemente el proceso de aprendizaje de una L2 (Brooks \& Swain, 2009; Kuiken \& Vedder, 2005; Nassaji \& Tian, 2010; Storch \& Wigglesworth, 2007).

La modalidad de trabajo de escritura colaborativa permite a los aprendientes de una L2 desarrollar gradualmente las habilidades necesarias para escribir textos coherentes de forma individual, autorregulada y autónoma, lo que se condice con hallazgos de investigaciones previas (Guzmán \& Rojas-Drummond, 2012; Storch \& Wigglesworth, 2007).

La evidencia demuestra que interactuar y comunicar ideas con otros miembros del grupo mientras se leen y escriben textos de tipo argumentativo permite que los aprendientes de una L2 participen en actividades de escritura más significativas aumentando su motivación, debido a que está constantemente apoyado y guiado por sus pares. Esto permite desarrollar la tarea de escritura dentro de un ambiente relajado y en un contexto comunicativo en que existe feedback permanente con relación al desempeño. Además, surge un conocimiento compartido con una audiencia real, la cual opina, participa, propone revisiones y edita. Todo esto permite mejorar la calidad de los textos dándole a los alumnos un sentido de pertenencia, lo que es coherente con lo planteado por diversos autores (Hirvela, 1999; Kessler, 2009; Kowal \& Swain, 1994; Leki, 1993; Rogoff, 2003; Rojas-Drummond et al., 2010; Storch, 2005; Swain \& Lapkin 1998).

Es importante recordar que al aplicar una metodología de escritura colaborativa en una L2 se deben aplicar los principios fundamentales de un aprendizaje basado en tareas, sustentado además en los principios teóricos de adquisición de segundas lenguas, como lo son la hipótesis de la interacción y la teoría sociocultural. El trabajo de escritura colaborativa sustentado en estos principios fundamentales se convierte en una estrategia que ayuda a reducir las dificultades lingüísticas en el proceso de adquisición del inglés, más aún, en el desarrollo de las habilidades de escritura. Esto debido a que en el contexto de nuestra realidad país ocurre particularmente que los estudiantes del sistema escolar, tanto de educación básica como educación media, tienen muy pocas oportunidades de práctica del inglés para el desarrollo de habilidades de producción escrita.

\section{REFERENCIAS BIBLIOGRÁFICAS}

Allen, N., Atkinson, D., Morgan, M., Moore, T., \& Snow, C. (1987). What experienced collaborators say about collaborative writing. Journal of Business and Technical Communication, 1(2), 70-90.

Brooks, L., \& Swain, M. (2009). Languaging in collaborative writing: Creation of and response to expertise. In A. Mackey \& C. Polio (Eds.), Multiple perspectives on interaction: Second language research in honor of Susan M. Gass (pp. 58-89). Nueva York, NY/Abingdon: Routledge.

Brown, J. S., \& Adler, R. P. (2008). Minds on fire: Open education, the long tail, and learning 2.0. EDUCAUSE Review, 43(1), 16-32.

Bruffee, K. A. (1993). Higher education, interdependence, and the authority of knowledge (1st ed.). Baltimore, MD: The Johns Hopkins University Press.

Bruffee, K. A. (1995). Sharing our toys: Cooperative learning versus collaborative learning. Change, 27(1), 12-18

Bruns, A., \& Humphreys, S. (2005). Wikis in teaching and assessment: The M/Cyclopedia project. 
Proceedings of the 2005 International Symposium on Wikis, 25-32.

Conner, N. (2008). Google Apps: The missing manual. Sebastopol, CA: Pogue Press/O'Reilly Media. Consejo de Europa. (2002). Marco común europeo de referencia para las lenguas: Aprendizaje, enseñanza, evaluación. Estrasburgo: Consejo de Europa/Ministerio de Educación/Grupo Anaya.

De Graaff, R., Jauregi, K., \& Nieuwenhuijsen, D. (2002). Collaborative learning in a virtual writing environment. Proceedings of the International Conference on Information and Communication Technologies in Education ICTE 2002, 302-306.

Derry, S. J. (1992). Beyond symbolic processing: Expanding horizons for educational psychology. Journal of Educational Psychology, 84(4), 413-418.

DiCamilla, F. J., \& Anton, M. (1997). Repetition in the collaborative discourse of L2 learners: A Vygotskian perspective. The Canadian Modern Language Review, 53(4), 609-633.

Dillenbourg, P. (1999). What do you mean by collaborative learning? In P. Dillenbourg (Ed.), Collaborative learning: Cognitive and computational approaches (pp. 1-19). Oxford: Elsevier.

Donato, R. (1994). Collective scaffolding in second language learning. En J. P. Lantolf \& G. Appel (Eds.), Vygotskian approaches to second language research (pp. 33-56). Westport, CT/Londres: Ablex Publishing.

Elola, I., \& Oskoz, A. (2010). Collaborative writing: Fostering foreign language and writing conventions development. Language Learning \& Technology, 14(3), 51-71.

Esteve, F. (2009). Bolonia y las TIC: De la docencia 1.0 al aprendizaje 2.0. La Cuestión Universitaria, 5, 59-68.

Fernández, J. M. (2009). Aprendiendo a escribir juntos: Multimodalidad, conocimiento y discurso. Monterrey: Comité Regional Norte de Cooperación con la UNESCO/Universidad Autónoma de Nuevo León.

Fisher, R., Myhill, D., Jones, S., \& Larkin, S. (2010). Using talk to support writing. Londres: SAGE.

García, J. (2010). Cambios en los estilos de aprendizaje inducidos por el uso de la web social. RED - Revista de Educación a Distancia, 22, 1-10.

Graham, C. R., \& Misanchuk, M. (2004). Computer-mediated learning groups: Benefits and challenges to using groupwork in online learning environments. In T. S. Roberts (Ed.). Online collaborative learning: Theory and practice (pp. 181-202). Hershey, PA: Information Science Publishing.

Guzmán, C. (2007). Taller de escritores: Una aproximación sociocultural para fortalecer la composición de textos en alumnos de primaria (Tesis de Maestría). Universidad Nacional Autónoma de México, Ciudad de México.

Guzmán, K., \& Rojas-Drummond, S. M. (2012). Escritura colaborativa en alumnos de primaria: Un modelo social de aprender juntos. Revista Mexicana de Investigación Educativa, 17(52), 217 245.

Hilton, S., \& Phillips, F. (2010). Instructor-assigned and student-selected groups: A view from inside. Issues in Accounting Education, 25(1), 15-33.

Hirvela, A. (1999). Collaborative writing instruction and communities of readers and writers. TESOL Journal, 8(2), 7-12.

Hoopingarner, D. (2009). Best practices in technology and language teaching. Language and Linguistics Compass, 3(1), 222-235.

Jauregi, K., Nieuwenhuijsen, D., \& de Graaff, R. (2003). A virtual writing environment for peer feedback in Spanish as a second language. En J. Piqué-Angordans, M. J. Esteve y M. L. GeaValor (Eds.), Internet in language for specific purposes and foreign language teaching (pp. 445456). Castellón de la Plana: Universitat Jaume I.

Johnson, D. W., \& Johnson, R. T. (1999). Learning together and alone: Cooperative, competitive, and individualistic learning (5th ed.). Boston, MA: Allyn \& Bacon.

Kessler, G. (2009). Student-initiated attention to form in wiki-based collaborative writing. Language Learning \& Technology, 13(1), 79-95. 
Kieser, A. L., \& Ortiz Golden, F. (2009). Using online office applications: Collaboration tools for learning. Distance Learning, 6(1), 41-46.

Klecker, B. M. (2003). Formative classroom assessment using cooperative groups: Vygotsky and random assignment. Journal of Instructional Psychology, 30(3), 216-219.

Kowal, M., \& Swain, M. (1994). Using collaborative language production tasks to promote students' language awareness. Language Awareness, 3(2), 73-93.

Kuiken, F., \& Vedder, I. (2002). Collaborative writing in L2: The effect of group interaction on text quality. In S. Ransdell \& M. L. Barbier (Eds.), New directions for research in L2 writing (pp. 169-188). Dordrecht: Kluwer Academic Publishers.

Kuiken, F., \& Vedder, I. (2005). Noticing and the role of interaction in promoting language learning. In A. Housen \& M. Pierrard (Eds.), Investigations in instructed second language acquisition (pp. 327-356). Berlín/Nueva York, NY: Mouton de Gruyter.

Lam, F. S., \& Pennington, M. C. (1995). The computer vs. the pen: A comparative study of word processing in a Hong Kong secondary classroom. Computer Assisted Language Learning, 8(1), 75-92.

Leki, I. (1993). Reciprocal themes in reading and writing. In J. Carson \& I. Leki (Eds.), Reading in the composition classroom: Second language perspectives (pp. 9-33). Boston, MA: Heinle \& Heinle.

Littleton, K., \& Hakkinen, K. P. (1999). Learning together: Understanding the processes of computerbased collaborative learning. In P. Dillenbourg (Ed.), Collaborative learning: Cognitive and computational approaches (pp. 20-31). Amsterdam: Pergamon.

Littleton, K., Miell, D., \& Faulkner, D. (Eds.). (2004). Learning to collaborate, collaborating to learn: Understanding and promoting educationally productive collaborative work. Nueva York, NY: Nova Science.

Lozano, A., Valdés, D. E., Sánchez, A. L., \& Esparza, E. (2011). Uso de Google Docs como herramienta de construcción colaborativa tomando en cuenta los estilos de aprendizaje. Actas de XII Encuentro de Virtual Educa, 1-17.

Meseke, C. A., Nafziger, R. E., \& Meseke, J. K. (2008). Student course performance and collaborative testing: A prospective follow-on study. Journal of Manipulative and Physiological Therapeutics, 31(8), 611-615.

Nassaji, H., y Tian, J. (2010). Collaborative and individual output tasks and their effects on learning English phrasal verbs. Language Teaching Research, 14(4), 397-419.

North, A. C., Linley, P. A., \& Hargreaves, D. J. (2000). Social loafing in a co-operative classroom task. Educational Psychology, 20(4), 389-392.

Oishi, L. (2007). Working together: Google Apps goes to school. Technology \& Learning, 27(9), 46-47.

Panitz, T. (1997). Collaborative versus cooperative learning: Comparing the two definitions helps understand the nature on interactive learning. Cooperative Learning and College Teaching, 8(2), 68-74.

Pérez, J. M. (2008). Teacher training curricula for media and information literacy: Background strategy paper international expert group meeting (Paris, June 2008). Recuperado de http://portal. unesco.org/ci/en/ev.php-URL_ID=27068\&URL_DO=DO_TOPIC\&URL_SECTION=201.html

Pico, L., \& Rodríguez, C. (2012). Trabajos colaborativos: Serie estrategias en el aula para el modelo 1 a 1. Buenos Aires: Ministerio de Educación.

Raimes, A. (1998). Teaching writing. Annual Review of Applied Linguistics, 18, 142-167.

Reid, J., \& Powers, J. (1993). Extending the benefits of small-group collaboration to the ESL writer. TESOL Journal, 2(4), 25-32.

Rogoff, B. (2003). The cultural nature of human development. Nueva York, NY: Oxford University Press.

Rojas-Drummond, S. M., Albarrán, C. D., \& Littleton, K. S. (2008). Collaboration, creativity and the 
co-construction of oral and written texts. Thinking Skills and Creativity, 3(3), 177-191.

Rojas-Drummond, S., Littleton, K., Hernández, F., \& Zúñiga, M. (2010). Dialogical interactions among peers in collaborative writing contexts. In K. Littleton \& C. Howe (Eds.), Educational dialogues: Understanding and promoting productive interaction (pp. 128-148). Abingdon/ Nueva York, NY: Routledge.

Rollinson, P. (2005). Using peer feedback in the ESL writing class. ELT Journal, 59(1), 23-30.

Shantz, C. U. (1987). Conflict between children. Child Development, 58, 283-305.

Storch, N. (1999). Are two heads better than one? Pair work and grammatical accuracy. System, 27(3), 363-374.

Storch, N. (2002). Patterns of interaction in ESL pair work. Language Learning, 52(1), 119-158.

Storch, N. (2005). Collaborative writing: Product, process, and students' reflections. Journal of Second Language Writing, 14(3), 153-173.

Storch, N. (2011). Collaborative writing in L2 contexts: Processes, outcomes, and future directions. Annual Review of Applied Linguistics, 31(1), 275-288.

Storch, N., \& Wigglesworth, G. (2007). Writing tasks: The effect of collaboration. In M. P. Garcia Mayo (Ed.), Investigating tasks in formal language learning (pp. 157-177). Clevedon: Multilingual Matters.

Swain, M., \& Lapkin, S. (1998). Interaction and second language learning: Two adolescent French immersion students working together. The Modern Language Journal, 82(3), 320-337.

Tharp, T. L. (2010). "Wiki, wiki, wiki-WHAT?" Assessing online collaborative writing. English Journal, 99(5), 40-46.

Tocalli-Beller, A. (2003). Cognitive conflict, disagreement and repetition in collaborative groups: Affective and social dimensions from an insider's perspective. The Canadian Modern Language Review, 60(2), 143-171.

van der Laan Smith, J., \& Spindle, R. M. (2007). The impact of group formation in a cooperative learning environment. Journal of Accounting Education, 25(4), 153-167.

Ware, P. D., \& O'Dowd, R. (2008). Peer feedback on language form in telecollaboration. Language Learning \& Technology, 12(1), 43-63. 